\title{
Gravitational dispersion in a torsional wave machine
}

\author{
Rafael de la Madrid,* Alejandro Gonzalez, and George M. Irwin \\ Department of Physics, Lamar University, Beaumont, Texas 77710
}

(Dated: August 20, 2014)

\begin{abstract}
We demonstrate that mechanical waves traveling in a torsional, mechanical wave machine exhibit dispersion due to gravity and the discreteness of the medium. We also show that although the dispersion due to discreteness is negligible, the dispersion due to gravity can be easily measured, and can be shown to disappear in a zero-gravity environment.
\end{abstract}




\section{INTRODUCTION}

Shive's torsional wave machine ${ }^{1}$ is one of the most widely used apparatuses for demonstrating mechanical waves in physics and engineering courses. Because most waves travel at speeds that are difficult to view, Shive created his wave machine to demonstrate slower transverse waves. Shive's machine can be used to demonstrate phenomena such as wave propagation, reflection and transmission at a boundary (fixed or free), constructive and destructive interference, standing waves and resonances, and impedance matching. ${ }^{1-7}$

According to Shive, the speed of the waves traveling along a torsional wave machine is a constant that depends on the stiffness of the wire and on the moment of inertia of the rods. However, Burgel ${ }^{2}$ realized that the speed of the waves traveling through a wave machine depends on the frequency, meaning a torsional wave machine is dispersive. After a thorough

analysis, Burgel concluded that the origin of the dispersion lies in the discreteness of the medium (in this case, the rods through which the waves propagate).

In the present paper, we re-examine Burgel's analysis and show that there are actually two sources of dispersion in a torsional wave machine. One source indeed originates from the discreteness of the medium, but a second, more important source originates from a restoring gravitational torque. We show that for practical purposes, the restoring gravitational torque is the only source of dispersion, whereas the effect of discreteness on the dispersion is negligible. In addition, we show that the gravitationally-induced dispersion is reduced in reduced gravity, and that it disappears in a zero-gravity environment.

The remainder of the paper is organized as follows. In Sec. II, we review the wave equation and the dispersion relation of a torsional wave machine. We also obtain the dispersion relation in the case that the discreteness of the rods is ignored and also when the gravitational field strength goes to zero. In Sec. III, we present the experimental procedure used to measure the harmonics of the wave machine, and in Sec. IV, we compare the experimental data with the theoretical predictions. Section V contains our conclusions.

Due to the simplicity of the experiments, the tractability of the theory, and the good agreement between theory and experiment, the results of our analysis can be easily adapted to an undergraduate laboratory on harmonics (normal modes), dispersion relations, and wave dispersion. 


\section{THEORY}

In our experiments, we use two PASCO wave machines. ${ }^{8}$ These wave machines have a central wire that is square shaped in cross section and runs through eleven supports (see Fig. 1). In one of the wave machines, the rods are 0.228-m long (the "short-rod machine"), whereas in the other one the rods are 0.456-m long (the "long-rod machine"). Each rod's center of mass is just below the wire, allowing the rods to be balanced horizontally in normal gravity (see Fig. 2). The long-rod machine has $N=71$ rods and a length of $L=0.89 \mathrm{~m}$, and the short-rod machine has $N=72$ rods and a length of $L=0.90 \mathrm{~m}$.

The theory describing motion in a wave machine is well known. ${ }^{1,2,5,6}$ Each rod of the machine oscillates as a pendulum around its horizontal, equilibrium position. As the torsion wire twists, the rods tilt away from their stable, equilibrium position, and two restoring torques act on the rods, one stemming from gravity and another one from the torsion of the wire. Let us denote by $j$ the rod number, with $j=1,2, \ldots, N$, where $N$ is the total number of rods. When the $j$ th rod is tilted, its weight produces a torque about the axis of rotation given by [see Fig. 2(b)]

$$
\tau_{\mathrm{g}}=-m g R \sin \left(\theta_{j}\right)
$$

where $m$ is the mass of the rod, $g$ is the gravitational field strength, $R$ is the rod's radius, and $\theta_{j}$ is the angular displacement from the horizontal, equilibrium position. The minus sign arises from the restoring nature of the gravitational torque. For small oscillations, the gravitational torque can be approximated by

$$
\tau_{\mathrm{g}}=-m g R \theta_{j}
$$

The torque on the $j$ th rod due to the twisting of the torsion wire can be written $\operatorname{as}^{2,5,6}$

$$
\tau_{\text {torsion }}=\kappa_{d}\left(\theta_{j-1}-\theta_{j}\right)+\kappa_{d}\left(\theta_{j+1}-\theta_{j}\right)
$$

where the constant $\kappa_{d}$ denotes the torsion constant of a section of the wire of length $d$, $d$ being the distance between two consecutive rods. By combining Eqs. (2) and (3) with Newton's second law for rotational motion, we obtain the equation of motion

$$
I_{\text {rod }}\left(\frac{\mathrm{d}^{2} \theta_{j}}{\mathrm{~d} t^{2}}\right)=-m g R \theta_{j}+\kappa_{d}\left(\theta_{j-1}-\theta_{j}\right)+\kappa_{d}\left(\theta_{j+1}-\theta_{j}\right),
$$


where $I_{\text {rod }}$ denotes the moment of inertia of one rod. Equation (4) holds for all but the end rods $(j=1$ and $j=N)$. The equation of motion of the end rods depends on the boundary conditions (either free or fixed), as discussed in Ref. 2.

When we send a pulse through a wave machine, the shape of the pulse does not change appreciably, and therefore the different frequencies making up the pulse travel at nearly the same speed $c$. Thus, for most applications, one can safely assume that the wave machine is non-dispersive. However, as first pointed out by Burgel, ${ }^{2}$ the wave machine is actually dispersive. As shown in Ref. 2, the dispersion relation associated with Eq. (4) is given by

$$
\omega^{2}=\omega_{\mathrm{p}}^{2}+4 \omega_{\mathrm{t}}^{2} \sin ^{2}\left(\frac{k d}{2}\right)
$$

where

$$
\omega_{\mathrm{t}}=\sqrt{\frac{\kappa_{d}}{I_{\mathrm{rod}}}}
$$

is the angular frequency of the torsional mode, and

$$
\omega_{\mathrm{p}}=\sqrt{\frac{m g R}{I_{\mathrm{rod}}}}
$$

is the angular frequency of the pendulum mode, in which the wave machine oscillates due to gravity alone $(\lambda=\infty, k=0)$.

In Eq. (5), there are two sources of dispersion. One source, due to the discreteness of the wave machine, is encoded in the separation $d$ between the rods. The second source, due to the gravitational restoring torque, is encoded in the pendulum-mode frequency. For the PASCO wave machines, the dispersion due to the discreteness of the rods is negligible, because the wavelength of the waves traveling on the machines is always much larger than $d$. Thus, $k d$ is always small and we can approximate $\sin (k d / 2) \approx k d / 2$. Within this approximation, Eq. (5) becomes

$$
\omega^{2} \approx \omega_{\mathrm{p}}^{2}+c^{2} k^{2}
$$

where

$$
c=\sqrt{\frac{\kappa_{L} L}{I / L}} \equiv \sqrt{\frac{\text { stiffness }}{\text { inertia }}}
$$

is the wave speed provided by Shive $^{1}$ (we provide an alternative derivation of the wave speed in Appendix B). In Eq. (9), $\kappa_{L}=\kappa_{d} d / L$ is the torsion constant of the entire wire of length $L$, and $I=N I_{\text {rod }}$ is the moment of inertia of the whole set of $N$ rods. 
The phase and group velocities can be easily calculated from Eq. (8), giving

$$
v_{\text {phase }}=\frac{\omega}{k} \approx \sqrt{\frac{\omega_{\mathrm{p}}^{2}}{k^{2}}+c^{2}}
$$

and

$$
v_{\text {group }}=\frac{\mathrm{d} \omega}{\mathrm{d} k} \approx \frac{c^{2} k}{\sqrt{\omega_{\mathrm{p}}^{2}+c^{2} k^{2}}}=\frac{c^{2}}{v_{\text {phase }}} .
$$

Because the phase and group velocities are different, the wave machine is dispersive even when we neglect the discreteness of the rods - the source of dispersion is the gravitational restoring torque. Interestingly, the gravitationally-induced dispersion disappears if we turn gravity off. To see this, we let $g$ go to zero in Eqs. (7) and (8) to get

$$
\omega \approx c k,
$$

which leads to identical expressions for the group and phase velocities:

$$
\begin{gathered}
v_{\text {phase }}=\frac{\omega}{k} \approx c, \\
v_{\text {group }}=\frac{\mathrm{d} \omega}{\mathrm{d} k} \approx c .
\end{gathered}
$$

Thus, when we neglect the discreteness of the rods and turn off gravity, the wave machine becomes non-dispersive.

Figure 3 shows a plot of the dispersion relations ( $\omega$ vs. $k$ ) from Eqs. (5), (8), and (12). From Fig. 3, we can see that the wave machines have three regions of dispersion. In the first region, which corresponds to low wave numbers, the exact dispersion relation given by Eq. (5) is well-approximated by Eq. (8). Thus, for low wave numbers the dispersion is due solely to the restoring gravitational torque. In this low- $k$ region, we find the pendulum mode (which corresponds to the $y$-intercept) and the lowest harmonics of the wave machine. In the second region, which corresponds to intermediate wave numbers, we have a dispersion-free region, since both Eqs. (5) and (8) are well approximated by the linear relationship given by Eq. (12). There is also a third, high- $k$ region of dispersion, where the exact dispersion relation [Eq. (5)] is not well-approximated by either Eq. (8) or Eq. (12). The dispersion in the high- $k$ region is due solely to the discreteness of the rods. In this high- $k$ region, there is a cut-off frequency, set by the spacing between rods in the wave machine. ${ }^{2}$ In the PASCO wave machines, this high- $k$ region is not attainable. 
Another way to see that the dispersion relation at low wave numbers is due solely to the gravitational torque and not to the discreteness of the rods is to determine the equation of motion and then find the dispersion relation when the rods are so close together that they can be considered a continuum. The resulting dispersion relation is, not surprisingly, given by Eq. (8) (see Appendix A for details). Thus, even when we ignore the discreteness of the rods from the start, we still have a gravitationally-induced dispersion at low wave numbers.

We note in passing that the dispersion relation in the wave machine [Eq. (8)] is mathematically equivalent to the dispersion relation of electromagnetic waves in a plasma at high frequencies (see Ref. 9). Such dispersion in a plasma is enhanced (diminished) whenever the charge of the particles in the plasma is increased (decreased), much like the dispersion in a wave machine is enhanced (diminished) when the gravitational field strength is increased (decreased).

\section{EXPERIMENTAL ARRANGEMENT}

An accelerometer (Vernier 3D-BTA) was mounted on one end of the torsion wire of the wave machine (see Fig. 1), and connected to a computer through an interface (Vernier LabQuest Mini). A wave pulse was then sent through the wave machine, and the accelerometer signal was converted into a frequency spectrum using a built-in Fast Fourier Transform (FFT) routine. A sample frequency spectrum is shown in Fig. 4. By clicking on the center of each peak of the frequency spectrum, we can measure the frequency of the harmonics excited by the pulse; the resulting frequencies are listed in Table I. The uncertainty in each frequency was obtained as the half-width at half-maximum of the peak in the frequency spectrum. It should be noted that different pulses may yield slightly different frequency spectra, and therefore slightly different harmonic frequencies, especially for the lowest harmonics. In such cases, the harmonic frequency can be taken as the average of the different measurements.

An alternative way to measure the harmonic frequencies is to excite the harmonics of the wave machine individually by way of an oscillator, as shown in Fig. 1(b). To accomplish this, we mounted a push/pull accessory (PASCO ME-8751) on an oscillator (PASCO ME-8750)

driven by a power supply. The push/pull accessory was attached to one of the end rods of the wave machine, on the opposite end to the accelerometer. The frequency of the oscillator 
was then increased incrementally until a resonance was obtained. Near the resonance, the frequency was finely tuned until the amplitude of the oscillation of the antinodes was as large as possible. The resulting frequency spectrum was just a sharp peak centered at the resonant (harmonic) frequency. By visually inspecting the number $n$ of antinodes of the harmonic, we were able to determine the wave number using a simple standing-wave analysis (see below). Except for the lowest harmonics, this method resulted in values that were within $3 \%$ of the values obtained using an FFT analysis of a wave pulse. For the lowest harmonics $(n \leq 4)$, the agreement was about $10 \%$ or better. The reason for the relatively poor agreement for the lowest harmonics is that we found it nearly impossible to excite only a single harmonic for such low frequencies. Thus, our measured frequencies for the pendulum mode and the lowest harmonics are the most unreliable.

The main advantage of using an oscillator to measure the frequencies is that students can see that each peak of the frequency spectrum corresponds to a specific standing wave mode. However, using the oscillator has some disadvantages. First, the data acquisition takes much longer than using an FFT analysis of a wave pulse. Second, we were only able to measure the first ten harmonics when using the oscillator, whereas the FFT analysis of a wave pulse allowed us to find over nineteen harmonics.

The wavelengths of the harmonics produced in a wave machine mimic exactly the wavelengths of the harmonics produced in a pipe. ${ }^{10}$ When both ends of the wave machine are free to oscillate (which corresponds to an open pipe), the wavelengths can be written

$$
\lambda_{n}=\frac{4 L}{n}, \quad n=2,4,6, \ldots \quad[\text { two free ends }]
$$

where $n$ is the number of antinodes of the wave machine. When we fix one end of the wave machine (which corresponds to a stopped pipe), the wavelengths can be written

$$
\lambda_{n}=\frac{4 L}{n}, \quad n=1,3,5, \ldots \quad \text { [single fixed end } .
$$

Thus, the wave numbers are given by ${ }^{11}$

$$
\left.k_{n}=n \frac{\pi}{2 L}, \quad n=2,4,6, \ldots \quad \text { [two free ends }\right]
$$

and

$$
\left.k_{n}=n \frac{\pi}{2 L}, \quad n=1,3,5, \ldots \quad \text { [single fixed end }\right] .
$$


In order to show the dependence of dispersion on gravity, we placed the wave machines in three different orientations. In the first arrangement the wave machines are positioned horizontally, so the gravitational field strength $g$ that appears in Eqs. (5) and (8) is given by $9.8 \mathrm{~m} / \mathrm{s}^{2}$ (one " $g$ "). In the second arrangement we tilt the wave machines by an angle $\alpha \simeq 60^{\circ}$, thereby reducing the effect of gravity by $\cos \left(60^{\circ}\right)=1 / 2$ (see Fig. 5 ). In this arrangement, we thus have a gravitational field strength of $4.90 \mathrm{~m} / \mathrm{s}$ (one-half $g$ ). Finally, in the third arrangement we hang the wave machines vertically, thereby eliminating the effect of gravity and producing a zero- $g$ environment. The measured frequencies for both wave machines in these three arrangements are listed in Table I.

It is important to note that because the wave machines are built to run horizontally, the friction between the rods and the supports increases quite a bit when the machines are tilted or hung. In order to minimize such friction, we connected the torsion wire of the wave machine to a fixed pole while the frame of the wave machine was immobilized by two supports. ${ }^{12}$

\section{COMPARISON BETWEEN THEORY AND EXPERIMENT}

In order to compare theory and experiment, we first need to determine the wave speeds and pendulum-mode frequencies. To determine these values, we perform linear regressions of the $\omega^{2}$ vs. $k^{2}$ data of Table I using Eq. (8) for the one- $g$ and the half- $g$ experiments. These regressions yield the wave speeds $c$ (the square root of the slope) and the pendulum-mode frequencies $\omega_{\mathrm{p}}$ (the square root of the $y$-intercept). We also perform linear regressions of the zero- $g$ data of Table I using Eq. (12). All of the fits had $R^{2}=0.9994$ or higher and are summarized in Table II. From this table, we see that the wave speed does not change appreciably as the effective gravitational field strength decreases, whereas the pendulummode frequency is seen to decrease.

In Figs. 6-8 we plot the experimental data from Table I (one $g$, one-half $g$, and zero $g$, respectively), along with the theoretical predictions given by either Eq. (8) or Eq. (12). These graphs include only the data from the long-rod wave machine, since the plots for the short-rod wave machine are almost identical (except that the frequencies of the short-rod wave machine are larger than those of the long-rod wave machine by a factor of about $\sqrt{8}$ ). In the graphs, $k$ is plotted in units of $\pi / 2 L$, so the wave numbers of the fixed-end harmonics 
are given by the odd integers (squares), whereas the wave numbers of the free-end harmonics are given by even integers (circles). The error bars are about the size of the data points and have not been included.

\section{A. Reducing the effect of gravity}

Figure 6 shows the data for the one- $g$ experiment. Here we plot the measured harmonic frequencies along with the theoretical dispersion relation [Eq. (8)] using the values of $c$ and $\omega_{\mathrm{p}}$ obtained from the linear regression. The inset shows the linear fit of the $\omega^{2}$ vs. $k^{2}$ data points. The agreement between theory and experiment shows that Eq. (8) fully accounts for the dispersion in the wave machines. Thus, for waves that can actually be created in the wave machines, the discreteness of the rods has no observable effect on the dispersion.

Figure 7 shows the data for the half- $g$ experiment. Again we plot the measured harmonic frequencies along with the dispersion relation given in Eq. (8). As in the one- $g$ experiment, the agreement between theory and experiment is reasonably good.

Figure 8 shows the data for the zero- $g$ experiment, where we plot the measured harmonic frequencies along with the dispersion relation of Eq. (12). The agreement between experiment and theory shows that the wave machines become non-dispersive in zero gravity.

In order to demonstrate more clearly how the gravitational dispersion in the wave machine decreases as we reduce the effect of gravity, Fig. 9 shows the pendulum mode and the first five harmonics of the long-rod wave machine for all three experiments. Here, we can see how the harmonic frequencies approach the straight line as gravity is reduced to zero.

\section{B. Ratio of wave speeds}

In the non-dispersive region, the theoretical wave speed in a torsional wave machine is given by Eq. (9). It is easy to show that because the long-machine rods are twice as long and twice as massive as the short-machine rods, and since their moments of inertia are $(1 / 12) m l^{2}$, where $m$ is the mass of a rod and $l$ its length, ${ }^{13}$ the speeds in the short and long wave machines are (theoretically) related by ${ }^{14}$

$$
\frac{c_{\text {short }}}{c_{\text {long }}}=\sqrt{8}=2.828 \text {. }
$$


Experimentally, we find that the ratio of the averages of the speeds is

$$
\frac{c_{\text {short }}}{c_{\text {long }}}=2.87 \pm 0.27,
$$

in good agreement with the theoretical result. It should be noted that this ratio is often reported simply as three. ${ }^{15}$

\section{CONCLUSIONS}

We have shown that dispersion in a torsional wave machine is due to gravity, and that such dispersion diminishes as gravity decreases. Experimentally, we reduced the effective gravitational strength by tilting the wave machine. In the limit when gravity goes to zero (in our case, when the wave machines are hung vertically), we showed that the gravitationallyinduced dispersion disappears and the wave machines becomes non-dispersive.

Our conclusions are restricted to the PASCO torsional wave machines used in our experiments (or other similar devices), and do not apply to all wave machines. For example, there is no gravitational dispersion in the wave machine used in Refs. 5 and 6 because this wave machine hangs from both ends rather than laying on supports. Thus, the dispersion in this machine results only from the discreteness of the rods. It should also be noted that in torsional wave machines of the type used in our experiment, the dispersion induced by the discreteness of the rods would be measurable if the rods were separated by a larger distance.

Finally, we would like to note that the two wave machines discussed here were flown in NASA's reduced gravity flight program in July 2012. The NASA flight allowed us to test the theory both at zero $g$ and at $1.8 \mathrm{~g}$. In the NASA flight, we excited the harmonics of the wave machine using an oscillator that was turned on and off for brief periods of time. The frequency of the oscillator was low, in order to excite mainly the lowest harmonics. When the oscillator was on, we had free-end boundary conditions, and when it was off, we had fixed-end boundary conditions.

Unfortunately, although our ground test of the experiment worked well, the results of the NASA flight do not agree conclusively with the results of the present paper. One possible reason is that, in the experiment in the NASA flight, the intervals during which the oscillator was turned on and off were not in sync with the periods of climbing $(1.8 \mathrm{~g})$ and descending (zero $g$ ) of the aircraft. A better procedure for this experiment would be the following: 
(1) manually excite a wave pulse at the beginning of a descending period of the aircraft and collect the FFT in zero $g$; (2) stop the motion of the wave machine at the end of the descending period; (3) excite a new wave pulse at the beginning of the climbing period and collect a new FFT in $1.8 g$; (4) repeat steps (1)-(3) for successive cycles of zero $g$ and $1.8 g$; (5) after the flight, obtain the harmonics in $1.8 \mathrm{~g}$ and zero $g$ from the FFTs.

\section{ACKNOWLEDGMENTS}

The authors would like to thank NASA for selecting the wave-machine experiment for its 2012 campaign of reduced gravity flights. The authors would also like to thank the LUV team (Nicholas Allen, Kirk Goza, Zach Jones, Jessica Plaia, Aleiya Samad, Aaron Weatherford, and Jacob Wright) for participating in the NASA flight. Special thanks are due to Jim Jordan and the Earth and Space Sciences Department of Lamar University for their technical support. The authors are also grateful to the journal's reviewers for their suggestions. One of the authors (RM) acknowledges financial support from Ministerio de Ciencia e Innovación of Spain under project TEC2011-24492.

\section{Appendix A: A continuum torsional wave machine}

In this appendix, we derive the equation of motion that describes the motion of a torsional wave machine in the continuum limit that the distance $d$ between the rods goes to zero. Using this equation of motion, we then derive the dispersion relation.

In a wave machine where the rods are packed together, let us consider a segment of length $\Delta x$. For small oscillations, Newton's second law of rotational motion for such a segment can be written as

$$
I_{\Delta x}\left(\frac{\partial^{2} \theta}{\partial t^{2}}\right)=-m_{\Delta x} g R \theta+\kappa_{\Delta x}\left(\theta_{x-\Delta x}-\theta\right)+\kappa_{\Delta x}\left(\theta_{x+\Delta x}-\theta\right)
$$

where $I_{\Delta x}$ is the moment of inertia of a segment of rods of length $\Delta x ; m_{\Delta x}$ is the mass of a segment of rods of length $\Delta x ; \theta, \theta_{x+\Delta x}$, and $\theta_{x-\Delta x}$ are the angular displacements from equilibrium at positions $x, x+\Delta x$, and $x-\Delta x$, respectively; $\kappa_{\Delta x}$ is the torsion constant of a section of wire of length $\Delta x$; and $R$ is the radius of a rod. If the rods are uniformly 
distributed, the mass of rods per unit of length is constant, and therefore

$$
\frac{M}{L}=\frac{m_{\Delta x}}{\Delta x}
$$

where $M$ is the mass of the whole set of rods, and $L$ is the length of the wave machine. Similarly, if the rods are uniformly distributed, the moment of inertia per unit length is constant, and therefore

$$
\frac{I}{L}=\frac{I_{\Delta x}}{\Delta x}
$$

where $I$ is the total moment of inertia of the whole set of rods. In addition, we have that

$$
\kappa_{\Delta x} \Delta x=\kappa_{L} L
$$

Substituting Eqs. (A2)-(A4) into Eq. (A1), and then multiplying and dividing the second term on the right-hand-side of Eq. (A1) by $\Delta x$, we obtain

$$
\Delta x \frac{I}{L}\left(\frac{\partial^{2} \theta}{\partial t^{2}}\right)=-\Delta x \frac{M}{L} g R \theta+\kappa_{L} L\left(\frac{\theta_{x+\Delta x}-\theta}{\Delta x}+\frac{\theta_{x-\Delta x}-\theta}{\Delta x}\right) .
$$

In the limit that $\Delta x$ is very small, we find that

$$
\Delta x \frac{I}{L}\left(\frac{\partial^{2} \theta}{\partial t^{2}}\right)=-\Delta x \frac{M}{L} g R \theta+\kappa_{L} L\left[\theta^{\prime}(x)-\theta^{\prime}(x-\Delta x)\right]
$$

Again, taking the limit that $\Delta x \rightarrow 0$, we arrive at the equation of motion

$$
\frac{I}{L}\left(\frac{\partial^{2} \theta}{\partial t^{2}}\right)=-\frac{M}{L} g R \theta+\kappa_{L} L \frac{\partial^{2} \theta}{\partial x^{2}} .
$$

By substituting a fixed-amplitude sinusoidal wave $\theta(t)=A e^{\mathrm{i}(k x-\omega t)}$ into Eq. (A7), we get the dispersion relation given by Eq. (8). Thus, not surprisingly, when we neglect the discreteness of the rods from the beginning, we find the same dispersion relation as was found when the discreteness of the rods was assumed to be negligible.

\section{Appendix B: Wave speed}

In Ref. 1, Shive states that the wave speed in a wave machine is given by Eq. (9). Such a wave speed can be derived by, for example, neglecting the restoring gravitational torque in Eq. (A7). Here, we provide an alternative derivation. Such a derivation can be viewed as the rotational analog of the derivation of the wave speed on a string (see, for example, Ref. 10, page 499). 
Let us consider the continuum version of the wave machine, neglecting the effect of the restoring gravitational torque. For this, let us imagine that we exert a torque $\tau$ on one of the ends of the wave machine. This torque will produce a disturbance that propagates with speed $c$. After a (small) time $\Delta t$, the disturbance has traveled a (small) distance $\Delta x=c \Delta t$ along the wave machine. The torque exerted on the wave machine is given by

$$
\tau=\kappa_{\Delta x} \Delta \theta
$$

Due to the torque, the angular momentum of a segment of the wave machine of length $\Delta x$ will change from 0 to $\ell$. On the one hand, $\ell$ is given by

$$
\ell=\tau \Delta t=\kappa_{\Delta x} \Delta \theta \Delta t
$$

and on the other it is given by

$$
\ell=I_{\Delta x} \omega=I_{\Delta x} \frac{\Delta \theta}{\Delta t} .
$$

Combining Eqs. (B2) and (B3) gives

$$
I_{\Delta x} \frac{\Delta \theta}{\Delta t}=\kappa_{\Delta x} \Delta \theta \Delta t
$$

and by canceling $\Delta \theta$ and multiplying by $\Delta x$ we get

$$
I_{\Delta x} \frac{\Delta x}{\Delta t}=\kappa_{\Delta x} \Delta x \Delta t
$$

Because the speed of the wave is $c=\Delta x / \Delta t$, and using $\kappa_{\Delta x} \Delta x=\kappa_{L} L$, we end up with

$$
I_{\Delta x} c=\kappa_{L} L \Delta t
$$

Since we are assuming that the wave machine is uniform, we have that $I_{\Delta x} / \Delta x=I / L$ and therefore

$$
\Delta x(I / L) c=\kappa_{L} L \Delta t
$$

Cross multiplication then yields

$$
\frac{\Delta x}{\Delta t} c=\frac{\kappa_{L} L}{I / L}
$$

or

$$
c^{2}=\frac{\kappa_{L} L}{I / L}
$$

which coincides with Eq. (9).

* Corresponding author's e-mail: rafael.delamadrid@lamar.edu 
1 J. N. Shive, Similarities in Wave Behavior (The Williams \& Wilkins Co. Series, Baltimore, Md., 1961).

2 B. A. Burgel, "Dispersion, Reflection, and Eigenfrequencies on the Wave Machine," Am. J. Phys. 35, 913-915 (1967).

3 T. B. Greenslade, "Apparatus for Natural Philosophy; Nineteenth Century Wave Machines," Phys. Teach. 18, 510-517 (1980).

4 J. Pizzo, "A Longitudinal Wave Machine," Phys. Teach. 27, 54-55 (1989).

5 K. D. Skeldon, J. E. Milne, A. I. Grant, and D. A. Palmer, "Making Waves: A Classroom Torsional Wave Machine (Part I)," Phys. Teach. 36, 392-398 (1998).

6 K. D. Skeldon, J. E. Milne, A. I. Grant, and D. A. Palmer, "Making Waves: A Classroom Torsional Wave Machine (Part II)," Phys. Teach. 36, 466-472 (1998).

7 J. D. Daffron, T. B. Greenslade, Jr., and J. Stille, "The Iowa Wave Machines," Phys. Teach. 48, 200-201 (2010).

8 PASCO, <http://www.pasco.com>.

9 J. D. Jackson, Classical Electrodynamics, 3rd ed. (Wiley, 1999), p. 313.

10 H. D. Young and R. A. Freedman, University Physics, 12th ed. (Pearson Addison-Wesley, 2008).

11 It should be noted that most textbooks denote the free-end wavelengths by $\lambda_{n}=2 L / n$, with $n=1,2,3, \ldots$, and the (single) fixed-end wavelengths by $\lambda_{n}=4 L / n$, with $n=1,3,5, \ldots$. We instead choose a quarter wavelength as the basic unit of length, so the free-end harmonics are associated with even integers, as in Eq. (17), whereas the (single) fixed-end harmonics are associated with odd integers, as in Eq. (18). Such a choice shows more clearly how the free-end and fixed-end harmonics alternate.

12 Further details on this experiment can be found in a supplementary video available at <https://www . youtube. com/watch?v=sA2Hbp8YsqE>.

13 The moment of inertia of each rod is actually $(1 / 12) m l^{2}+(5 / 4) m R^{2}$, where $R$ is the radius of the rod. However, because $R \ll l$, the moment of inertia is well approximated by $(1 / 12) m l^{2}$.

14 For simplicity, we have assumed that the lengths of the short and the long wave machines are the same, since taking into account the actual lengths $(0.89 \mathrm{~m}$ and $0.90 \mathrm{~m})$ barely changes the ratio given in Eq. (19).

15 See, for example, the Harvard Natural Sciences Lecture Demonstrations website, <http://www.fas.harvard.edu/ scdiroff/lds/OscillationsWaves/ShiveWaveMachine/ 


\section{ShiveWaveMachine.html>.}

TABLE I. Frequencies and wave numbers of all the harmonics. All frequencies are in $\mathrm{Hz}$ and the error for each experiment is given in parentheses. Equations (17) and (18) were used to calculate the wave numbers; the length $L$ of the long-rod and short-rod wave machines are, respectively, $0.890 \pm 0.004 \mathrm{~m}$ and $0.900 \pm 0.004 \mathrm{~m}$ (the uncertainty being equal to the diameter of one rod).

\begin{tabular}{|c|c|c|c|c|c|c|}
\hline & \multicolumn{6}{|c|}{ Harmonic frequencies $(\mathrm{Hz})$} \\
\hline & \multicolumn{3}{|c|}{ Long-rod machine } & \multicolumn{3}{|c|}{ Short-rod machine } \\
\hline$n \quad k_{n}$ & $\begin{array}{c}\text { one } g \\
( \pm 0.03)\end{array}$ & $\begin{array}{l}\text { one-half } g \\
\quad( \pm 0.05)\end{array}$ & $\begin{array}{c}\text { zero } g \\
( \pm 0.08)\end{array}$ & $\begin{array}{l}\text { one } g \\
( \pm 0.1)\end{array}$ & $\begin{array}{l}\text { one-half } g \\
\quad( \pm 0.4)\end{array}$ & $\begin{array}{l}\text { zero } g \\
( \pm 0.7)\end{array}$ \\
\hline $0 \quad 0$ & 0.22 & 0.15 & 0 & 0.44 & 0.29 & 0 \\
\hline $1 \frac{\pi}{2 L}$ & 0.27 & 0.24 & 0.15 & 0.59 & 0.54 & 0.44 \\
\hline $2 \quad \frac{\pi}{L}$ & 0.35 & 0.34 & 0.29 & 0.93 & 0.88 & 0.83 \\
\hline $3 \quad \frac{3 \pi}{2 L}$ & 0.46 & 0.44 & 0.42 & 1.17 & 1.22 & 1.17 \\
\hline $4 \quad \frac{2 \pi}{L}$ & 0.60 & 0.59 & 0.56 & 1.66 & 1.66 & 1.56 \\
\hline $5 \quad \frac{5 \pi}{2 L}$ & 0.72 & 0.73 & 0.68 & 1.95 & 1.98 & 1.96 \\
\hline $6 \quad \frac{3 \pi}{L}$ & 0.85 & 0.83 & 0.81 & 2.39 & 2.39 & 2.34 \\
\hline $7 \quad \frac{7 \pi}{2 L}$ & 0.98 & 0.98 & 0.95 & 2.73 & 2.73 & 2.73 \\
\hline $8 \frac{4 \pi}{L}$ & 1.11 & 1.12 & 1.10 & 3.17 & 3.13 & 3.11 \\
\hline $9 \quad \frac{9 \pi}{2 L}$ & 1.26 & 1.22 & 1.22 & 3.52 & 3.52 & 3.52 \\
\hline $10 \frac{5 \pi}{L}$ & 1.37 & 1.37 & 1.37 & 3.91 & 3.91 & 3.83 \\
\hline $11 \frac{11 \pi}{2 L}$ & 1.51 & 1.51 & 1.51 & 4.30 & 4.25 & 4.25 \\
\hline $12 \frac{6 \pi}{L}$ & 1.64 & 1.61 & 1.61 & 4.64 & 4.67 & 4.61 \\
\hline $13 \frac{13 \pi}{2 L}$ & 1.78 & 1.76 & 1.76 & 5.08 & 5.08 & 5.08 \\
\hline $14 \frac{7 \pi}{L}$ & 1.90 & 1.90 & 1.88 & 5.42 & 5.47 & 5.42 \\
\hline $15 \frac{15 \pi}{2 L}$ & 2.05 & 2.05 & 2.05 & 5.86 & 5.76 & 5.86 \\
\hline $16 \frac{8 \pi}{L}$ & 2.17 & 2.15 & 2.15 & 6.20 & 6.20 & 6.20 \\
\hline $17 \frac{17 \pi}{2 L}$ & 2.32 & 2.29 & 2.32 & 6.64 & 6.59 & 6.54 \\
\hline $18 \frac{9 \pi}{L}$ & 2.44 & 2.44 & 2.42 & 6.93 & 6.98 & 6.88 \\
\hline
\end{tabular}


TABLE II. Results of linear regressions of the Table I data as described in the text. All fits had $R^{2}=0.9994$ or higher.

\begin{tabular}{c|cc|cc}
\hline \hline \multicolumn{2}{c}{ Long-rod wave machine } & \multicolumn{2}{c}{ Short-rod wave machine } \\
\hline Effective $g$ & $c(\mathrm{~m} / \mathrm{s})$ & $\omega_{\mathrm{p}}(\mathrm{rad} / \mathrm{s})$ & $c(\mathrm{~m} / \mathrm{s})$ & $\omega_{\mathrm{p}}(\mathrm{rad} / \mathrm{s})$ \\
\hline \multirow{2}{*}{ one $g$} & $0.4867 \pm 0.0007$ & $1.6 \pm 0.1$ & $1.393 \pm 0.003$ & $3.1 \pm 0.6$ \\
one-half $g$ & $0.484 \pm 0.001$ & $1.4 \pm 0.2$ & $1.392 \pm 0.002$ & $2.9 \pm 0.5$ \\
zero $g$ & $0.479 \pm 0.002$ & $0.08 \pm 0.04$ & $1.382 \pm 0.005$ & $0.23 \pm 0.09$ \\
\hline \hline
\end{tabular}

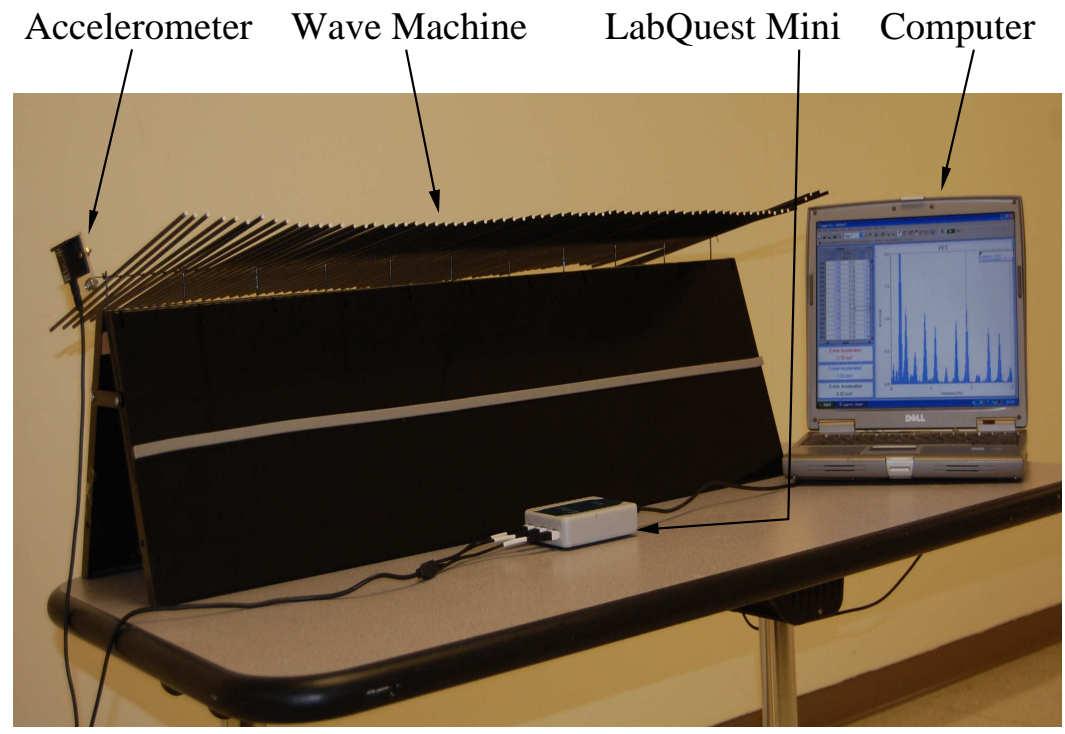

(a)

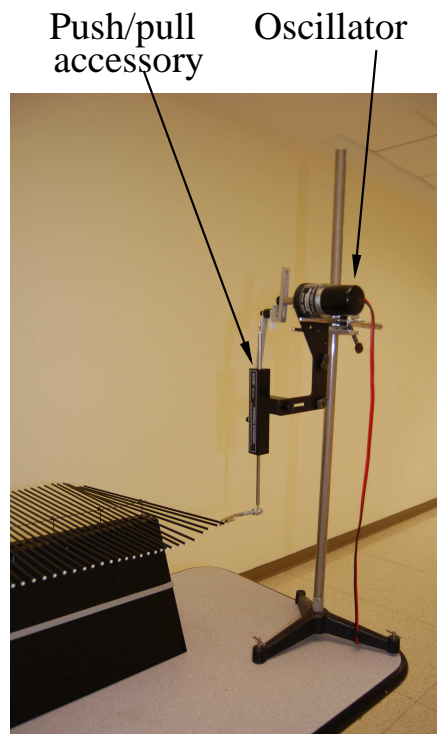

(b)

FIG. 1. (a) Experimental apparatus. (b) The oscillator drives the wave machine through PASCO's push/pull accessory, which is attached to one of the end rods. 


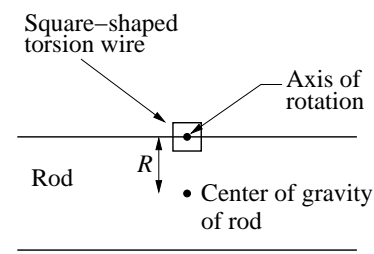

(a)

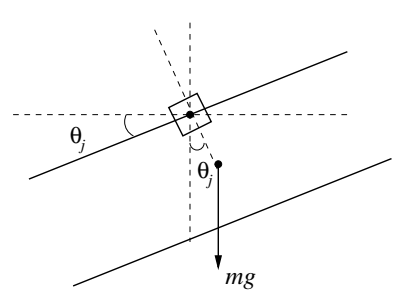

(b)

FIG. 2. Rod in (a) equilibrium position, and (b) tilted by an angle $\theta_{j}$. Because the center-of-gravity of the rod is below the axis of rotation, the weight $m g$ of the rod produces a restoring gravitational torque of magnitude $m g R \sin \left(\theta_{j}\right)$.

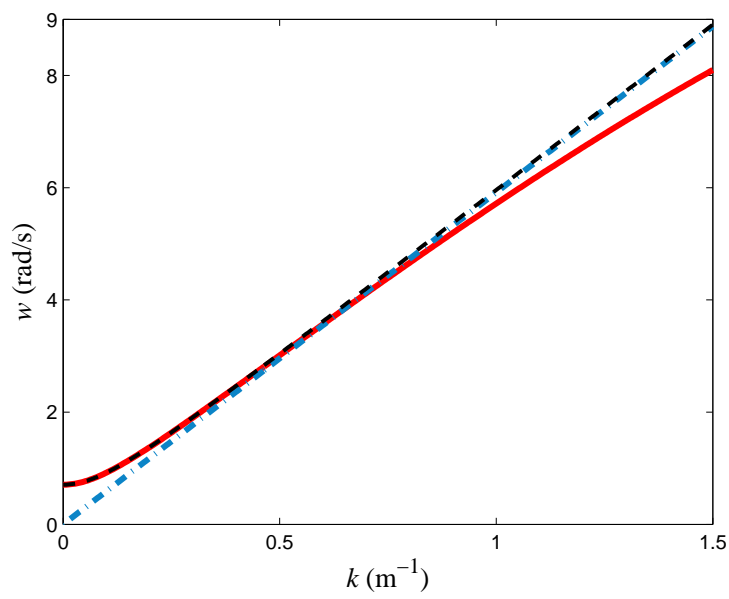

FIG. 3. Plots of the dispersion relations: Eq. (5) (red, solid line), Eq. (8) (black, vertically-dashed line), and Eq. (12) (blue, dash-dot line). The plots correspond to $c=\sqrt{35} \mathrm{~m} / \mathrm{s}, \omega_{\mathrm{p}}=0.5 \mathrm{rad} / \mathrm{s}$, $\omega_{\mathrm{t}}=1 \mathrm{rad} / \mathrm{s}$, and $d=1 \mathrm{~m}$. 


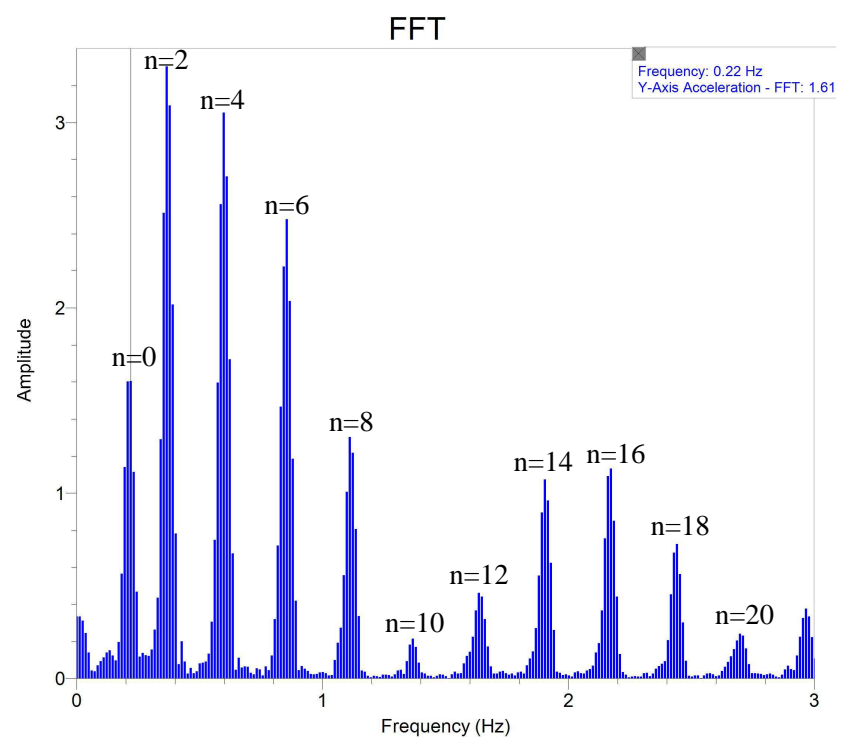

FIG. 4. FFT frequency spectrum of a wave pulse sent through the long-rod wave machine in a one- $g$ environment with free ends. By clicking on the center of each peak, the harmonic frequency is displayed in an inset at the top right corner. The pendulum mode $(n=0)$ corresponds to the peak of lowest frequency $(0.22 \mathrm{~Hz})$.

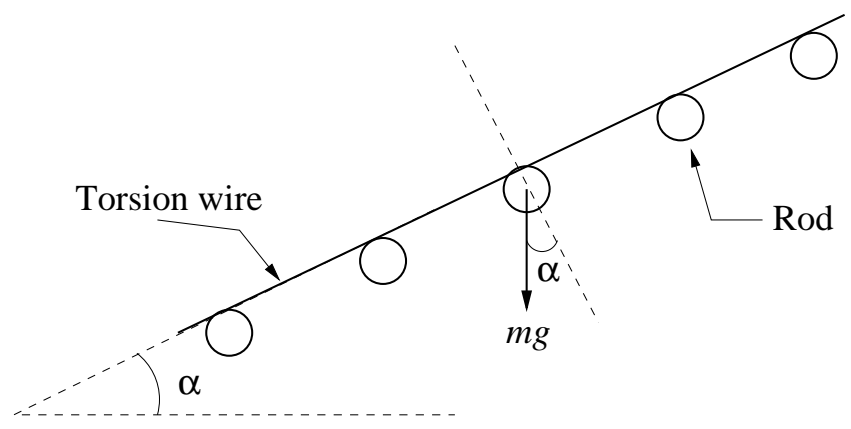

FIG. 5. Wave machine tilted by an angle $\alpha$. The component of each rod's weight parallel to the torsion wire $(m g \sin \alpha)$ does not produce any torque. Only the component of the rod's weight perpendicular to the torsion wire $(m g \cos \alpha)$ produces a torque. Hence, tilting the wave machine by an angle $\alpha$ effectively reduces the strength of gravity to $g \cos \alpha$. 


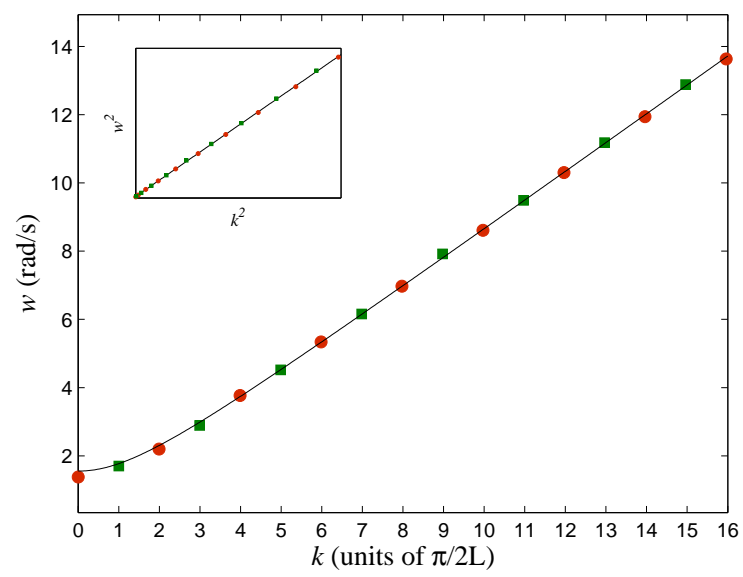

FIG. 6. Harmonics of the long-rod wave machine in a one- $g$ environment, and comparison with the dispersion relation given by Eq. (8) (solid curve). The red circles represent the free-end harmonics and the green squares represent the fixed-end harmonics. The inset displays the linear regression of the $\omega^{2}$ vs. $k^{2}$ data points.

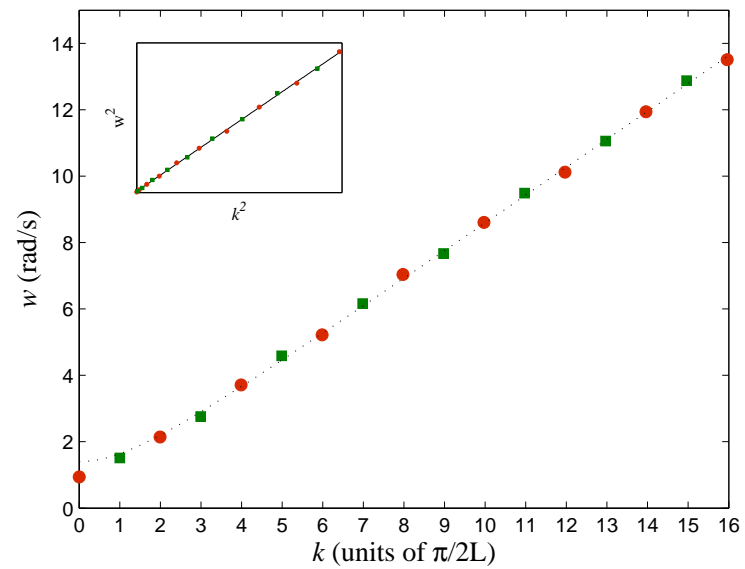

FIG. 7. Harmonics of the long-rod wave machine in a half- $g$ environment, and comparison with the dispersion relation given by Eq. (8) (dotted curve). The red circles represent the free-end harmonics, and the green squares represent the fixed-end harmonics. The inset displays the linear regression of the $\omega^{2}$ vs. $k^{2}$ data points. 


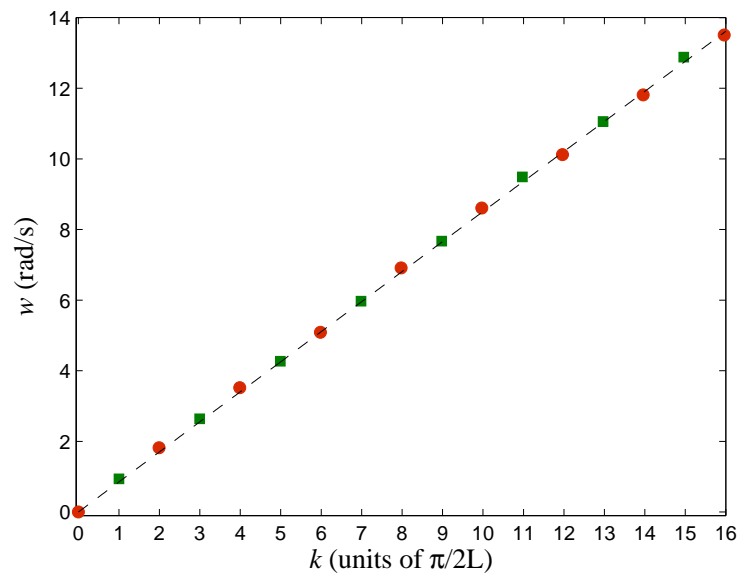

FIG. 8. Harmonics of the long-rod wave machine in a zero- $g$ environment, and comparison with the dispersion relation given by Eq. (12) (dashed line). The red circles represent the free-end harmonics, and the green squares represent the fixed-end harmonics.

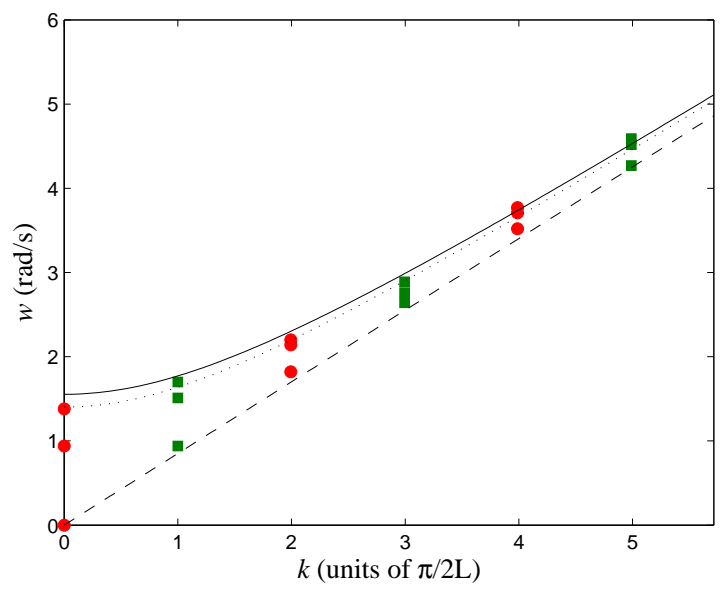

FIG. 9. Dependence of the dispersion on gravity in the long-rod wave machine in the low- $k$ region. The solid curve corresponds to one $g$, the dotted curve corresponds to one-half $g$, and the dashed line corresponds to zero- $g$. 